\title{
Lack of replication of genetic associations with human longevity
}

\author{
Valeria Novelli · Chiara Viviani Anselmi · Roberta Roncarati · \\ Guia Guffanti - Alberto Malovini - Giulio Piluso - Annibale Alessandro Puca
}

Received: 6 September 2007 / Accepted: 4 November 2007/Published online: 23 November 2007

(C) Springer Science+Business Media B.V. 2007

\begin{abstract}
The exceptional longevity of centenarians is due in part to inherited genetic factors, as deduced from data that show that first degree relatives of centenarians live longer and have reduced overall mortality. In recent years, a number of groups have performed genetic association studies on long-living individuals (LLI) and young controls to identify alleles that are either positively or negatively selected in the centenarian population as consequence of a demographic pressure. Many of the reported studies have shown genetic loci associated with longevity. Of these, with the exception of APOE, none have been convincingly reproduced. We validated our populations by typing the APOE locus. In addition, we used 749 American Caucasian LLI, organized in two independent tiers and 355 American Caucasian controls in the attempt to replicate previously published findings. We tested Klotho (KL)-VS variant (rs952706), Cholesteryl Ester Transfer Protein (CETP) I405V (rs5882), Paraoxonase 1 (PON1)
\end{abstract}

V. Novelli · C. Viviani Anselmi - R. Roncarati .

A. Malovini · A. A. Puca ( $\triangle)$

Genetic Department, IRCCS Multimedica, via Milanese

300, Sesto S. Giovanni (Milan) 20099, Italy

e-mail: puca@longevita.org

G. Guffanti

University of Milano, Milano, Italy

G. Piluso

Second University of Naples, Napoli, Italy
Q192R (rs662), Apolipoprotein C-III (APOC3) -641C/A (rs2542052), Microsomal Transfer Protein (MTP) -493G/T (rs2866164) and apolipoprotein E (APOE) $\varepsilon 2$ and $\varepsilon 4$ isoforms, (rs7412 and rs429358) haplotypes respectively. Our results show that, at present, except for APOE, none of the selected genes show association with longevity if carefully tested in a large cohort of LLI and their controls, pointing to the need of larger populations for case-control studies in extreme longevity.

Keywords Association studies ·

Long living individuals · Longevity

\section{Introduction}

The frequency of centenarians in the industrialized world is 1 in 10,000 people and this prevalence is rapidly changing, approaching 1 in 5,000 born in the near future (Perls 2006). The recent reduced mortality in western countries is partly a result of recent changes in food availability and diet (Cordain et al. 2005) and to other environmental changes, such as reduced exposure to infection and consequent reduction in inflammation due the discovery of antibiotics and improvement of health care. The sum of these changes has pushed the female life expectancy in industrialized countries to rise 3 months/year for 160 years by scoring a 4 decade increase in life expectancy in the last 16 decades (Oeppen and 
Vaupel 2002). In addition, many other observations point to environmental factors as modulators of human longevity, such as the relationship between lower burden of sickness during childhood (expressed as reduced child mortality), a person's birth order in the family (as index of young age of the mother) and survival to extreme old age (Gavrilova NS and Gavrilov LA 2007).

On the other hand, the role of genetics in exceptional longevity is suggested by the dramatic reduction of centenarian sibling mortality levels (Perls et al. 2002). We recognize that among the phenotype traits analyzed until now, extreme longevity is apparently the most complex of all, being influenced by individual disease susceptibility and the rate of aging. In addition, we do not know which ages should be adopted to observe demographic pressure that is the loss of detrimental alleles and the enrichment of protective ones as the population age. For example, we would not expect to capture loci that influence longevity acting at a very old age if the majority of the individuals chosen for the case arm were still young. We know that genetic case control studies are influenced by many potential bias that are intrinsic in the design. False positive results are mainly due to stratification (admixture in the studied populations), lack of appropriate corrections for multiple testing (due to unpublished negative results) and lack of replications. False negatives are influenced by small sample size and the young ages in the case study. In addition, the identification of extreme longevity genetic determinants relies upon the ability to find the right genes to test among the components of biological pathways related to aging. Following APOE association with longevity, the most scrutinized pathway has been lipid metabolism. Other strategies include the investigation of human homo$\log$ of longevity genes in lower eukaryotes such as DAF genes in C. Elegans (Christensen et al. 2006) and the SIR2 gene in S. Cerevisiae (Gartenberg 2000).

Some non-synonimous SNPs of the genes Insulin Growth Factor-1 Receptor (IGF-1R), apolipoprotein B (APOB), APOC3, CETP, Angiotensin I Converting Enzyme (ACE), Interleukin 6 (IL6), PON1, Sirtuin 3 (SIRT3), Klotho and many others have also been tested and shown to have associations with extreme longevity. However, these associations have not been successfully reproduced. Of the few dozen polymorphisms tested so far by the scientific community, the only locus consistently associated with longevity is the haplotype that determines APOE isoforms $\varepsilon 2$ and $\varepsilon 4$ (Lewis and Brunner. 2004). Apolipoprotein $\mathrm{E}$ is a polymorphic protein involved in transport and redistribution of lipids in various tissues (Panza et al. 2003). The two isoforms $\varepsilon 2$ and $\varepsilon 4$ were previously associated with susceptibility to cardiovascular and Alzheimer disease and subsequently were also associated with extreme longevity (Schachter et al. 1994).

In this work, we attempted to replicate previous reported associations using: (1) APOE as an internal positive control to validate our populations; (2) populations that in the previous experiments did not show evidence of stratification (data unpublished); (3) two large independent cohorts of LLI.

Among the several polymorphisms that have been previously associated with longevity, we have chosen the association studies that in our opinion needed further clarification.

The SNPs tested in this paper are $-493 \mathrm{G} / \mathrm{T}$ MTP, APOE $\varepsilon 2$ (TT) and $\varepsilon 4$ (CC) haplotypes, CETP I405V, KL-VS, PON1 Q192R and -641 C/A APOC3.

MTP assembles very low density lipoprotein (VLDL) in the liver and regulate the chilomicrons intestinal absorption. A sib-pair analysis strategy was adopted to identify a locus at D4S1564 (Puca et al. 2001) that was subsequently investigated with haplotype based associations on 875 polymorphisms that identified a $-493 \mathrm{G} / \mathrm{T}$ MTP variant. This variant showed a consistent association in a second group of 250 centenarians and 250 controls selected to minimize the mahalanobis distance with respect to the cases to avoid stratification (Geesaman et al. 2003) (Table 1). As for many other studies, the MTP association has not been reproduced in Caucasian European populations (Nebel et al. 2005; Beekman et al. 2006, (Table 1).

Mice homozygous for severely hypomorphic alleles of the Klotho gene showed features of accelerated aging (Kuro-o et al. 1997), while transgenic mice over-expressing the gene were long-living (Kurosu et al. 2005), making this gene an appealing candidate for human genetic association studies. The heterozygous Klotho variant KL-VS stretch (that contain six polymorphisms) in a Bohemian Czech population was increased in the elderly (protective effect) while the homozygous form was decreased 
Table 1 Allele frequencies in reported studies of microsomal triglycerid transfer protein

\begin{tabular}{|c|c|c|c|c|c|c|c|c|c|}
\hline \multirow[t]{2}{*}{ MTP (-493 G/T) (Ref) } & \multirow[t]{2}{*}{ Nationality } & \multirow[t]{2}{*}{$\mathrm{LLI}^{\mathrm{b}}$} & \multirow[t]{2}{*}{$\mathrm{Age}^{\mathrm{c}}$} & \multirow[t]{2}{*}{ Offspring } & \multirow[t]{2}{*}{ Controls } & \multirow[t]{2}{*}{ Age } & \multicolumn{2}{|c|}{ Allele frequency ${ }^{\mathrm{d}}(\%)$} & \multirow[t]{2}{*}{$P$ value } \\
\hline & & & & & & & G & $\mathrm{C}$ & \\
\hline \multirow[t]{3}{*}{ Geesaman et al. (2003) } & U.S. Caucasian & 175 & 100.8 & & 183 & 38.6 & 26 vs 33 & 74 vs 67 & 0.039 \\
\hline & U.S. Caucasian & 244 & 100.8 & & 231 & 38.6 & 24 vs 32 & 76 vs 68 & 0.029 \\
\hline & French & 559 & 103.1 & & 552 & 51.2 & 25 vs 26 & 75 vs 74 & 0.54 \\
\hline \multirow[t]{2}{*}{ Nebel et al. (2005) } & German & 370 & 102 & & 540 & 67 & 28 vs 25 & 72 vs 75 & 0.28 \\
\hline & German & 1033 & 98.2 & & 540 & 67 & 26 vs 25 & 73 vs 75 & 0.34 \\
\hline \multirow[t]{3}{*}{ Beekman et al. (2006) } & Caucasian & & & 525 & 251 & 59 & 27 vs 24 & 73 vs 76 & 0.41 \\
\hline & Caucasian & 379 & 94 & & 251 & 59 & 26 vs 24 & 74 vs 76 & 0.60 \\
\hline & Caucasian & 655 & 87 & & 244 & 31 & 24.9 vs 24 & 75.1 vs 76 & 0.64 \\
\hline
\end{tabular}

Abbreviations: ${ }^{\mathrm{a}}$ : reference; ${ }^{\mathrm{b}}$ : long living individuals; ${ }^{\mathrm{c}}$ : refer to mean age; ${ }^{\mathrm{d}}$ : \%centenarians vs $\%$ controls

(detrimental effect) (Arking et al. 2002). The protective effect of the heterozygous state was not corroborated in a second population from the Baltimore WHAS and BWIS studies, stratified by race, while the protective role of the homozygous for VS genotype was confirmed (Arking et al. 2002).

A follow-up study on Ashkenazi-Jewish populations replicated the heterozygous advantage but not the homozygous disadvantage (Arking et al. 2005) (Table 2).

Individuals with exceptional longevity have shown significantly larger low density lipoprotein (LDL) and high density lipoprotein (HDL) particle sizes (Barzilai et al.2003) and consequently CETP, which determines lipid particle size (Arai et al. 2000), was investigated in the above mentioned Ashkenazi-Jewish populations. Among the few SNPs tested, the homozygous GG genotype of the CETP I405V polymorphism (that correspond to $\mathrm{VV}$ ), increased in centenarians and centenarian offspring (Barzilai et al. 2003). A follow up study on European centenarians did not replicate the association (Cellini et al. 2005) (Table 2).

APOC3 is a major component of very LDLs and a minor component of HDL. In a recent study, Ashkenazi-Jewish populations were genotyped for 66 SNPs in 33 candidate genes related to cardiovascular diseases. Among these, APOC3 -641CC genotypes were more frequent among long-living individuals. The CC carriers had lower prevalence of hypertension and greater insulin sensitivity (Atzmon et al. 2006) (Table 2).

Q192R variant of PON1 was selected for its role in regulating LDL oxidation. This polymorphism showed a controversial association with longevity, being sometimes reported as increased (protective) in centenarian populations, and sometimes decreased (detrimental) (Rea et al. 2004) (Table 2).

The results reported in this study hopefully clarify the need of using large populations to avoid false positives and identify true associations with longevity, like APOE.

\section{Methods}

Subjects

Group I: 381 US LLI (309 females and 79 males, mean age 101.7 years [age range 93-111; Confidence interval \pm 9.3$]$ ); group II: 368 US LLI (174 females and 194 males, mean age 95.9 years [age range 93-105; Confidence interval \pm 8.7$]$ ) and 355 anonymous controls, self-identified as "Caucasian" and $<35$ years of age (191 females and 164 males, mean age 27.4 years [age range 0-35; Confidence interval \pm 7.6$]$ ). LLI data collected included health and socio-demographic histories, proof of age, usually in the form of a birth certificate, a three-generation pedigree, and measures to assess functional independence and cognitive status.

The subjects were selected among the general population by different strategies, contacted by mail or telephonically, and properly consented.

The institutional review board granted approval for this study and all subjects involved signed an informed consent form. This study was conducted in accordance with the ethical principles that have their origins in the Declaration of Helsinki. Individuals were identified and recruited by a variety of methods 


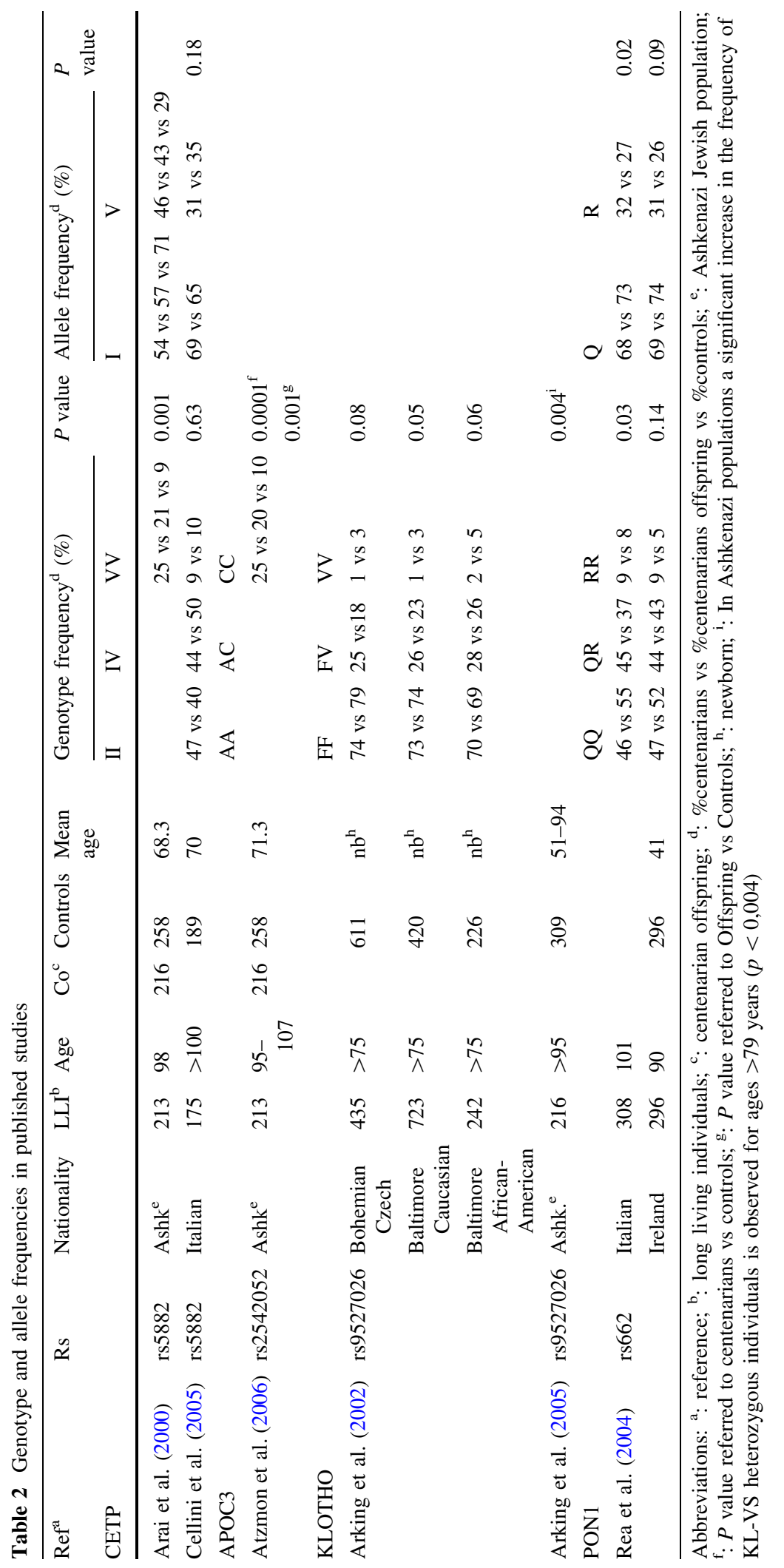


including institutional web sites, direct mailings, and advertisements in newspapers targeting potential participants or organizations involved with the aging community.

\section{DNA isolation and genotyping}

Centenarian and control DNA was isolated from whole blood using a Qiamp DNA blood Midikit (Qiagen) according to the manufacturer's protocol.

Genotyping was performed using Taqman Assay (Applied Biosystems). PCRs and and post-PCR fluorescence measurements were carried out on an ABI7900 (Applied Biosystems). For genotyping, cluster plots were made of the fluorescent labels using SDS software (v2.2 Applied Biosystems). Spots falling outside a cluster were labelled as undetermined. Standard quality control procedures were applied such us duplications of the DNA extractions and comparisons of the new aliquots with plated DNA by SNP typing.

\section{Statistical analysis}

The chi-square test was performed using the statistical package Stata ve 9.1 for a comparison of the allele and genotype frequencies between the groups.

We used a subroutine GENHW to estimate allele, genotype frequencies and disequilibrium coefficients which performs asymptotic Hardy-Weinberg (HW) equilibrium tests.

For the case-control genetic studies was used GENCC that calculates the $p$-value and the confidence interval (95\%) of the odds ratio. A $p$-value less than 0.05 was considered the threshold for statistical significance. The subroutine TABULATE was used to produce one-way tables of frequency counts along with measures of association. The haplotype association test between cases and controls was performed using Haploview 3.2 software. (http://www. broad.mit.edu/personal/jcbarret/haploview/index.php) (Barret et al. 2005).

\section{Results}

In the present study we have genotyped 749 LLI (divided in two groups of samples) and 355 controls from USA for seven SNP in the genes supposedly associated with longevity.

The analysis of APOE $\varepsilon 2$ (TT haplotype, Cys112Cys158) and $\varepsilon 4$ (CC haplotype, Arg112-Arg158) isoforms (rs7412 and rs429358) showed, as expected, that isoform $\varepsilon 2$ is enriched and isoform $\varepsilon 4$ is depleted in centenarians compared to controls (Table 3 ).

-493G/T MTP polymorphism was genotyped using the perfectly correlated SNP rs2866164 (Geesaman et al. 2003). The first comparison (LLI group I versus controls) shows a statistically significant decrease in the frequency of the minor allele and the genotype GG in centenarians compared to control population, similar to our previous report (Geesaman et al. 2003) (Table 4). In the second comparison (LLI group II versus controls) the association did not approach significance. Due to other reports that show a difference in control allele frequency, we genotyped an additional 156 controls (mean age 30.2 years). By including these controls the association disappeared (Table 4).

The homozygous GG (that corresponds to VV) for CETP I405V (rs5882) shows no differences in frequency among cases and controls (Table 5). This is contrary to the original data published (Arai et al. 2000), but is in accordance with a subsequent attempt to reproduce the results in Italian populations (Cellini et al. 2005) (Table 2).

KL-VS variant (rs952706), PON1 Q192R (rs662), and -641C/A APOC3 (rs2542052) showed the same distributions of allele and genotype frequencies between cases and controls (Table 5), not supporting previous reports (Table 2). In addition, we combined the analysis of group I and II (Table 5) to see if a modest effect could be detected by a larger population set. None of the association tests supported the original findings, including -493G/T MTP, and despite a nominal $\mathrm{p}$ value of $p=0.04(p=0.08 / 2$ being the hypothesis tested one-way: cases minor allele frequency $<$ control minor allele frequency),

Table 3 APOE $\varepsilon 4$ haplotype frequencies

\begin{tabular}{lllll}
\hline & $\begin{array}{l}\text { ApoE } \\
\text { isoforms }\end{array}$ & $\begin{array}{l}\text { LLI }^{\mathrm{a}} \text { vs controls } \\
\text { frequencies }\end{array}$ & $\begin{array}{l}\text { Chi- } \\
\text { square }\end{array}$ & $P$ value \\
\hline US group I & $\varepsilon 4$ & 0.055 vs 0.118 & 19.419 & 0.0000105 \\
US group II & $\varepsilon 4$ & 0.067 vs 0.122 & 12.968 & 0.0003 \\
\hline
\end{tabular}

Abbreviations: ${ }^{\text {a}}$ : long living individuals 


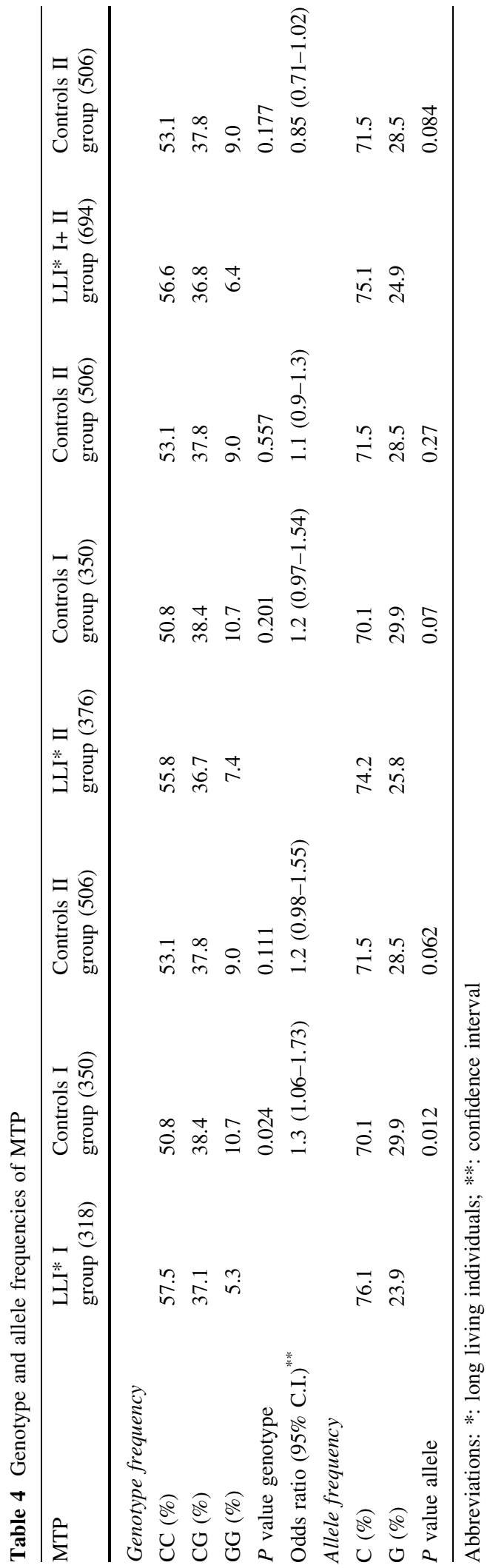

the association test did not pass the level of significance after Bonferroni correction for multiple testing.

\section{Discussion}

Many case control studies for exceptional longevity have been published in the recent years, and, with the exception of APOE, none of the initial findings have been consistently reproduced. In an attempt to shed light on longevity variants, we selected some of the SNPs previously reported to be associated with longevity. To avoid false positive associations we decided to genotype two tiers of centenarians and one of controls, to minimize eventual admixture among the centenarians. To address the problem of false negative results (lack of power), we have run the study on large set of samples (380) with average ages of cases above 95 years to capture eventual alleles that confer advantage or disadvantage at very old ages. The results of our analysis show a consistent association of APOE $\varepsilon 4$ with longevity while the analysis of Klotho KL-VS variant (rs952706), CETP I405V (rs5882), PON1 Q192R (rs662), -641C/A APOC3 (rs2542052) and -493G/T MTP (rs2866164) showed no associations with exceptional longevity.

To be noted, we had to run additional 156 controls for $-493 \mathrm{G} / \mathrm{T}$ MTP to disentangle the incongruent reports of the other groups that showed no association.

Overall, these results indicate that, for the exception of APOE, some of the previous attempts to identify genes influencing longevity are not replicable.

An explanation on why so many findings are not replicated by other studies could be that reported associations, due to stratification by age between cases and controls, instead of pointing to longevity enabling polymorphisms, select polymorphisms that have changed in frequency in younger generations due to recent immigration. This could be especially true in the case of polymorphisms that show a great difference in allele frequencies among ethnicities and so more sensitive to undetected mild stratification. It has to be said that, despite the above mentioned reasonable theories, our initial analysis of 450 nonagenarians and 450 young controls with 317 thousand SNPs did not show any stratification 
Table 5 Genotype and allele frequencies of PON1, Klotho, CETP and ApoC3

\begin{tabular}{|c|c|c|c|c|}
\hline & \multicolumn{2}{|l|}{$\mathrm{LLI}^{\mathrm{a}}$} & \multirow{2}{*}{$\begin{array}{l}\text { LLI }^{\mathrm{a}} \\
\text { Group I + II }\end{array}$} & \multirow[t]{2}{*}{ Controls } \\
\hline & Group I & Group II & & \\
\hline PON1 (Q192R) & $(350)^{\mathrm{b}}$ & $(360)^{\mathrm{b}}$ & $(710)^{\mathrm{b}}$ & $(351)^{\mathrm{b}}$ \\
\hline GG $(\%)$ & 9.8 & 10.2 & 10 & 11 \\
\hline $\mathrm{AG}(\%)$ & 40.8 & 39.7 & 40.2 & 39.1 \\
\hline $\mathrm{AA}(\%)$ & 49.3 & 50 & 49.6 & 49.8 \\
\hline$P$ value genotype & 0.477 & 0.933 & 0.849 & \\
\hline $\mathrm{G}(\%)$ & 30.3 & 30.1 & 30.2 & 30.7 \\
\hline $\mathrm{A}(\%)$ & 69.7 & 69.9 & 69.8 & 69.3 \\
\hline$P$ value allele & 0.861 & 0.816 & 0.814 & \\
\hline Odds ratio $\left(95 \%\right.$ C.I. $\left.^{c}\right)$ & $1.0(0.81-1.27)$ & $0.97(0.77-1.21)$ & $0.97(0.80-1.18)$ & \\
\hline KLOTHO (KL-VS) & $(345)^{\mathrm{b}}$ & $(363)^{b}$ & $(708)^{b}$ & $(332)^{b}$ \\
\hline GG $(\%)$ & 67.8 & 76.4 & 73 & 72.5 \\
\hline $\mathrm{AG}(\%)$ & 28.1 & 21.7 & 24 & 25.6 \\
\hline $\mathrm{AA}(\%)$ & 4.1 & 1.8 & 3 & 1.8 \\
\hline$P$ value genotype & 0.146 & 0.235 & 0.350 & \\
\hline $\mathrm{G}(\%)$ & 81.9 & 88.1 & 85.1 & 85.4 \\
\hline $\mathrm{A}(\%)$ & 18.1 & 11.9 & 14.9 & 14.6 \\
\hline$P$ value allele & 0.081 & 0.2437 & 0.721 & \\
\hline Odds ratio $\left(95 \%\right.$ C.I. $\left.^{c}\right)$ & $1.29(0.96-1.72)$ & $0.79(0.58-1.08)$ & $1.04(0.80-1.36)$ & \\
\hline CETP (I405V) & $(361)^{b}$ & $(361)^{\mathrm{b}}$ & $(722)^{b}$ & $(348)^{b}$ \\
\hline GG $(\%)$ & 9.1 & 11.3 & 10.2 & 11.4 \\
\hline $\mathrm{AG}(\%)$ & 40.4 & 41.2 & 40.8 & 47.4 \\
\hline AA $(\%)$ & 50.4 & 47.4 & 48.9 & 41 \\
\hline$P$ value genotype & 0.041 & 0.19 & 0.050 & \\
\hline $\mathrm{G}(\%)$ & 29.4 & 32 & 30.6 & 35.2 \\
\hline $\mathrm{A}(\%)$ & 70.6 & 68 & 69.4 & 64.8 \\
\hline$P$ value allele & 0.018 & 0.188 & 0.0335 & \\
\hline Odds ratio $\left(95 \%\right.$ C.I. $\left.{ }^{\mathrm{c}}\right)$ & $1.3(1.04-1.63)$ & $1.15(0.93-1.43)$ & $0.81(0.67-0.98)$ & \\
\hline APOC3 (-641 C/A $)$ & $(356)^{\mathrm{b}}$ & $(357)^{\mathrm{b}}$ & $(713)^{\mathrm{b}}$ & $(354)^{\mathrm{b}}$ \\
\hline $\mathrm{CC}(\%)$ & 11.5 & 13.7 & 12.7 & 16.1 \\
\hline $\mathrm{AC}(\%)$ & 48 & 46.5 & 47.3 & 44 \\
\hline AA $(\%)$ & 40.4 & 39.6 & 39.9 & 39.9 \\
\hline$P$ value genotype & 0.189 & 0.638 & 0.289 & \\
\hline A $(\%)$ & 64.5 & 63 & 63.6 & 61.9 \\
\hline $\mathrm{C}(\%)$ & 35.5 & 37 & 36.4 & 38.1 \\
\hline$P$ value allele & 0.309 & 0.672 & 0.4103 & \\
\hline Odds ratio $\left(95 \%\right.$ C.I. $\left.{ }^{\mathrm{c}}\right)$ & $0.89(0.72-1.10)$ & $0.95(0.77-1.18)$ & $0.92(0.76-1.11)$ & \\
\hline
\end{tabular}

Abbreviations: ${ }^{\mathrm{a}}$ : long living individuals ${ }^{\mathrm{b}}$ : number of samples; ${ }^{\mathrm{c}}$ : confidence interval

(unpublished data), in agreement with other studies (Cardon and Palmer 2003). Our data confirm the difficulty in replicating previous associations with human longevity. The use of small sample sizes and over interpretation of marginal results in the initial findings, the interpopulation heterogeneity of geneenvironment and gene-gene interactions and the subtle differences in the selected phenotypes are possible explanations for such discrepancies (Cardon and Palmer 2003). 
Acknowledgments This study was supported by grants of Italian Ministry of University and Research (MIUR); Contract grant number: FIRB RBIN04X9XE. The authors wish to thank Elixir Pharmaceuticals Inc. for providing the collection of centenarians and controls.

\section{References}

Arai T, Tsukada T, Murase T, Matsumoto K (2000) Particle size analysis of high density lipoproteins in patients with genetic cholesteryl ester transfer protein deficiency. Clin Chim Acta 301(1-2):103-117

Arking DE, Krebsova A, Macek M Sr, Macek M Jr, Arking A, Mian IS, Fried L, Hamosh A, Dey S, McIntosh I, Dietz HC (2002) Association of human aging with a functional variant of klotho. Proc Natl Acad Sci USA 99(2):856-861

Arking DE, Atzmon G, Arking A, Barzilai N, Dietz HC (2005) Association between a functional variant of the KLOTHO gene and high-density lipoprotein cholesterol, blood pressure, stroke, and longevity. Circ Res 96(4):412-418

Atzmon G, Rincon M, Schechter CB, Shuldiner AR, Lipton RB, Bergman A, Barzilai N (2006) Lipoprotein genotype and conserved pathway for exceptional longevity in humans. PLoS Biol 4(4):e113

Barrett JC, Fry B, Maller J, Daly MJ (2005) Haploview: analysis and visualization of $\mathrm{LD}$ and haplotype maps. Bioinformatics 99:100-110

Barzilai N, Atzmon G, Schechter C, Schaefer EJ, Cupples AL, Lipton R, Cheng S, Shuldiner AR (2003) Unique lipoprotein phenotype and genotype associated with exceptional longevity. JAMA 290(15):2030-2040

Beekman M, Blauw GJ, Houwing-Duistermaat JJ, Brandt BW, Westendorp RG, Slagboom PE (2006) Chromosome 4q25, microsomal transfer protein gene, and human longevity: novel data and a meta-analysis of association studies. $\mathbf{J}$ Gerontol A Biol Sci Med Sci 61(4):355-362

Cardon LR, Palmer LJ (2003) Population stratification and spurious allelic association. Lancet 361(9357):598-604. Review

Cellini E, Nacmias B, Olivieri F, Ortenzi L, Tedde A, Bagnoli S, Petruzzi C, Franceschi C, Sorbi S (2005) Cholesteryl ester transfer protein (CETP) I405V polymorphism and longevity in Italian centenarians. Mech Ageing Dev 126(6-7):826-828

Christensen K, Johnson TE, Vaupel JW (2006) The quest for genetic determinants of human longevity: challenges and insights. Nat Rev Genet 7(6):436-448

Cordain L, Eaton SB, Sebastian A, Mann N, Lindeberg S, Watkins BA, O'Keefe JH, Brand-Miller J (2005) Origins and evolution of the Western diet: health implications for the 21st century. Am J Clin Nutr 81(2):341-354

Gartenberg MR (2000) The Sir proteins of Saccharomyces cerevisiae: mediators of transcriptional silencing and much more. Curr Opin Microbiol 3(2):132-7
Gavrilova NS and Gavrilov LA (2007) North Am Actuar J 11(1): 49-67

Geesaman BJ, Benson E, Brewster SJ, Kunkel LM, Blanché H, Thomas G, Perls TT, Daly MJ, Puca AA (2003) Haplotype-based identification of a microsomal transfer protein marker associated with the human lifespan. Proc Natl Acad Sci USA 100(24):14115-14120

Kuro-o M, Matsumura Y, Aizawa H, Kawaguchi H, Suga T, Utsugi T, Ohyama Y, Kurabayashi M, Kaname T, Kume E, Iwasaki H, Iida A, Shiraki-Iida T, Nishikawa S, Nagai R, Nabeshima YI (1997) Mutation of the mouse klotho gene leads to a syndrome resembling ageing. Nature 390(6655):45-51

Kurosu H, Yamamoto M, Clark JD, Pastor JV, Nandi A, Gurnani P, McGuinness OP, Chikuda H, Yamaguchi M, Kawaguchi H, Shimomura I, Takayama Y, Herz J, Kahn CR, Rosenblatt KP, Kuro-o M (2005) Suppression of aging in mice by the hormone Klotho. Science 309(5742):1829-1833

Lewis SJ, Brunner EJ (2004) Methodological problems in genetic association studies of longevity - the apolipoprotein E gene as an example. Int J Epidemiol 33(5):962-970

Nebel A, Croucher PJ, Stiegeler R, Nikolaus S, Krawczak M, Schreiber S (2005) No association between microsomal triglyceride transfer protein (MTP) haplotype and longevity in humans. Proc Natl Acad Sci USA 102(22): 7906-7909

Oeppen J, Vaupel JW (2002) Broken limits to life expectancy. Science 296(5570):1029-1031

Panza F, Solfrizzi V, Colacicco AM, Basile AM, D'Introno A, Capurso C, Sabba M, Capurso S, Capurso A (2003) Apolipoprotein E (APOE) polymorphism influences serum APOE levels in Alzheimer's disease patients and centenarians. Neuroreport 14(4):605-608

Perls TT (2006) The different paths to 100. Am J Clin Nutr 83(2):484S-487S

Perls TT, Wilmoth J, Levenson R, Drinkwater M, Cohen M, Bogan H, Joyce E, Brewster S, Kunkel L, Puca A (2002) Life-long sustained mortality advantage of siblings of centenarians. Proc Natl Acad Sci USA 99:8442-8447

Puca AA, Daly MJ, Brewster SJ, Matise TC, Barrett J, SheaDrinkwater M, Kang S, Joyce E, Nicoli J, Benson E, Kunkel LM, Perls T (2001) A genome-wide scan for linkage to human exceptional longevity identifies a locus on chromosome 4. Proc Natl Acad Sci USA 98(18): 10505-10508

Rea IM, McKeown PP, McMaster D, Young IS, Patterson C, Savage MJ, Belton C, Marchegiani F, Olivieri F, Bonafe M, Franceschi C (2004) Paraoxonase polymorphisms PON1 192 and 55 and longevity in Italian centenarians and Irish nonagenarians. A pooled analysis. Exp Gerontol 39(4):629-635

Schachter F, Faure-Delanef L, Guenot F, Rouger H, Froguel P, Lesueur-Ginot L, Cohen D (1994) Genetic associations with human longevity at the APOE and ACE loci. Nat Genet 6(1):29-32 